\title{
A Surgical Case of Bilateral Pulmonary Metastases from Thyroid Follicular Cancer
}

\author{
Motohisa Kuwahara1 $^{*}$, Masato Iwami1, Masae Mano1, Toshihiko Moroga' ${ }^{2}$, Akinori Iwasaki² \\ ${ }^{1}$ Division of Surgery, Saiseikai Futsukaichi Hospital, Fukuoka, Japan \\ ${ }^{2}$ Departement of Chest Surgery, Fukuoka University Hospital, Fukuoka, Japan \\ Email: ${ }^{*}$ KHC03152@nifty.com
}

Received 24 September 2015; accepted 18 October 2015; published 21 October 2015

Copyright $@ 2015$ by authors and Scientific Research Publishing Inc.

This work is licensed under the Creative Commons Attribution International License (CC BY).

http://creativecommons.org/licenses/by/4.0/

c) (i) Open Access

\begin{abstract}
An 80-year-old woman underwent a bilateral lung resection for metastases originating from follicular thyroid cancer. The resection was performed 30 years after right hemithyroidectomy to remove the follicular adenoma. Chest X-ray revealed a $30-\mathrm{mm}$ mass shadow in the right lower lung filed. Chest computed tomography revealed a 32-mm mass shadow in right lung segment 10 (S10) and a 15-mm nodular shadow in left S10. Another partial lung resection of left S10 and a right lower lobectomy were performed 3 months later. Although rarely performed, resection of bilateral pulmonary metastases arising from follicular thyroid cancer was conducted for this patient.
\end{abstract}

\section{Keywords}

Thyroid Follicular Cancer, Pulmonary Metastases, Metastasectomy

\section{Introduction}

Thyroid carcinomas are uncommon malignancies. They account for approximately $2 \%$ of all new cancer cases [1], but rank first among endocrine tumors in terms of frequency and mortality. Follicular cancers account for $10 \%$ $20 \%$ of all thyroid malignancies. They tend to occur more frequently in older patients. The bones, lungs and central nervous system are the most common sites of metastasis of thyroid follicular carcinoma. Treatment of distant metastases depends on the nature, extent, and radioiodine avidity of the disease [2]. Total thyroidectomy followed by radioactive iodine and suppressive treatment with L-thyroxin has been proposed for patients with synchronous distant metastasis. Patients with radioiodine refractory disease receive a kinase inhibitor during molecular target therapy or systemic chemotherapy.

We described an 80-year-old woman who underwent bilateral lung resections for metastases originating from

"Corresponding author.

How to cite this paper: Kuwahara, M., Iwami, M., Mano, M., Moroga, T. and Iwasaki, A. (2015) A Surgical Case of Bilateral Pulmonary Metastases from Thyroid Follicular Cancer. Surgical Science, 6, 442-445.

http://dx.doi.org/10.4236/ss.2015.610063 
follicular thyroid cancer. The resections were performed 30 years after a right hemithyroidectomy procedure implemented to treat follicular adenoma.

\section{Case Report}

An 80-years-old woman underwent right hemithyroidectomy for probable follicular adenoma of the right thyroid gland 30 years previously. No regional lymph node swellings and distant metastatic tumors were found during the 2-year follow-up period.

She presented at our hospital bilateral lower limbs swelling. Chest X-ray showed a mass shadow in the right lower lung field (Figure 1(a)). Chest computed tomography (CT) revealed a 32-mm mass shadow in right lung segment 10 (S10) and a 15-mm nodular shadow in left S10 (Figure 1(b) and Figure 1(c)). Bronchoscopy revealed no abnormal findings. Bronchoscopic biopsy specimens of the right S10 showed no specific findings.

She was admitted for thoracoscopic resection of the left lung nodule. She had no history of drinking or smoking and her family histories were unremarkable. She had surgery scars at the cervix and the bilateral varicose veins of the lower extremity. Thoracoscopic partial lung resection of the left S10 was performed. On cut section, the tumor had a yellow to tan homogenous appearance. Histologically, the tumor formed follicular structures similar to the thyroid gland. The pathological diagnosis was pulmonary metastases from follicular thyroid cancer.

The values of TSH $(2.9 \mu \mathrm{IU} / \mathrm{ml})$ and free T4 $(1.05 \mathrm{ng} / \mathrm{dl})$ were within normal limits. Accumulation of fluorodexyglucose on positron emission tomography was not shown, and a bone scintigram revealed nothing unusual. Resection of the remnant thyroid gland and radioisotope therapy were recommended to the patient, but she refused.

Three months later, right lower lung lobectomy and lymph node dissection were performed because the follow-up chest CT scan revealed that lobular lymph node enlargement was present (Figure 2(a)). Similar to the previous findings in the left lung, histopathologic findings of the resected tumor revealed thyroid gland tissue with follicular projection (Figure 2(b)). The diagnosis was metastases from thyroid follicular carcinoma. No evidence of metastatic tumor tissue was seen in the dissected lymph nodes. Resection of remnant thyroid gland and radioisotope therapy were again recommended, but she refused. After the pulmonary resections, we provided follow-up care at our out-patient clinic. The patient had no signs of recurrence at the 3-year follow-up appointment.

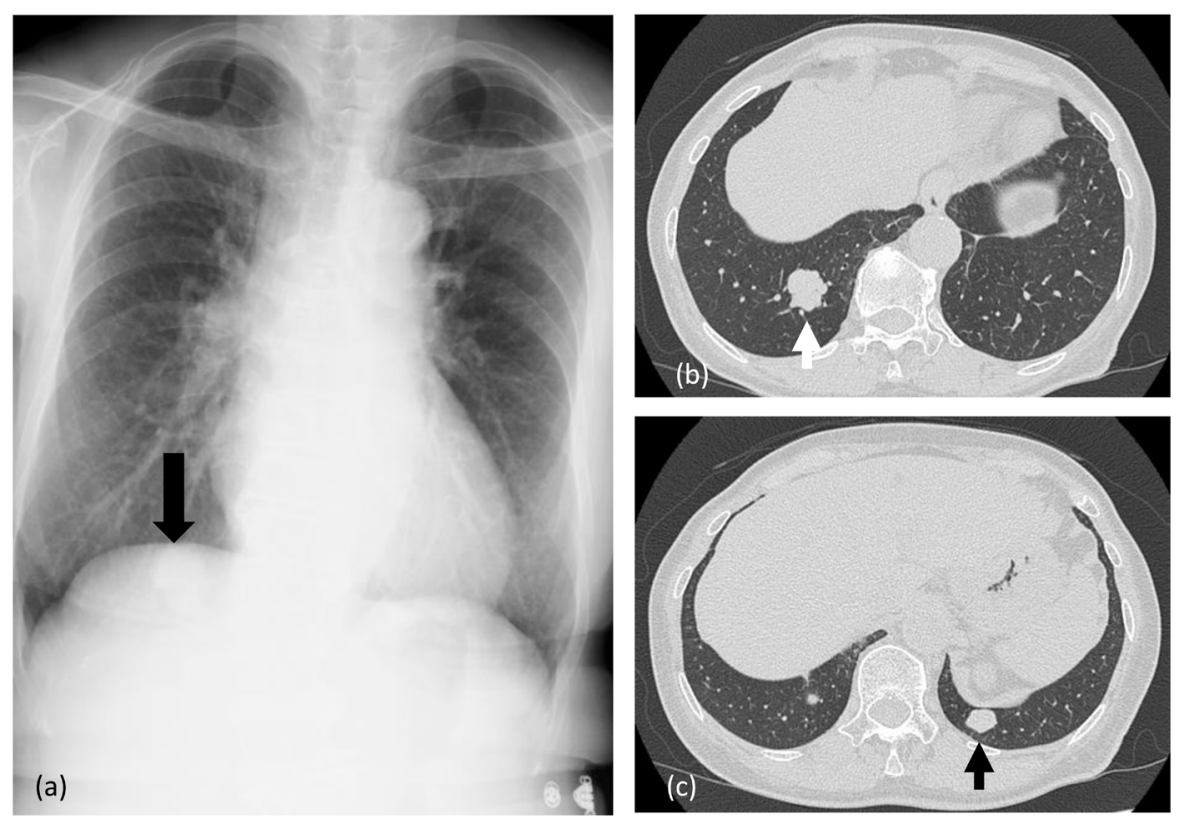

Figure 1. (a) The chest X-ray showed a mass shadow in the right lower lung fields; (b) Chest computed tomography (CT) revealed a 32-mm mass shadow in right lung segment 10; (c) CT revealed and 15-mm nodular shadow in left S10. 

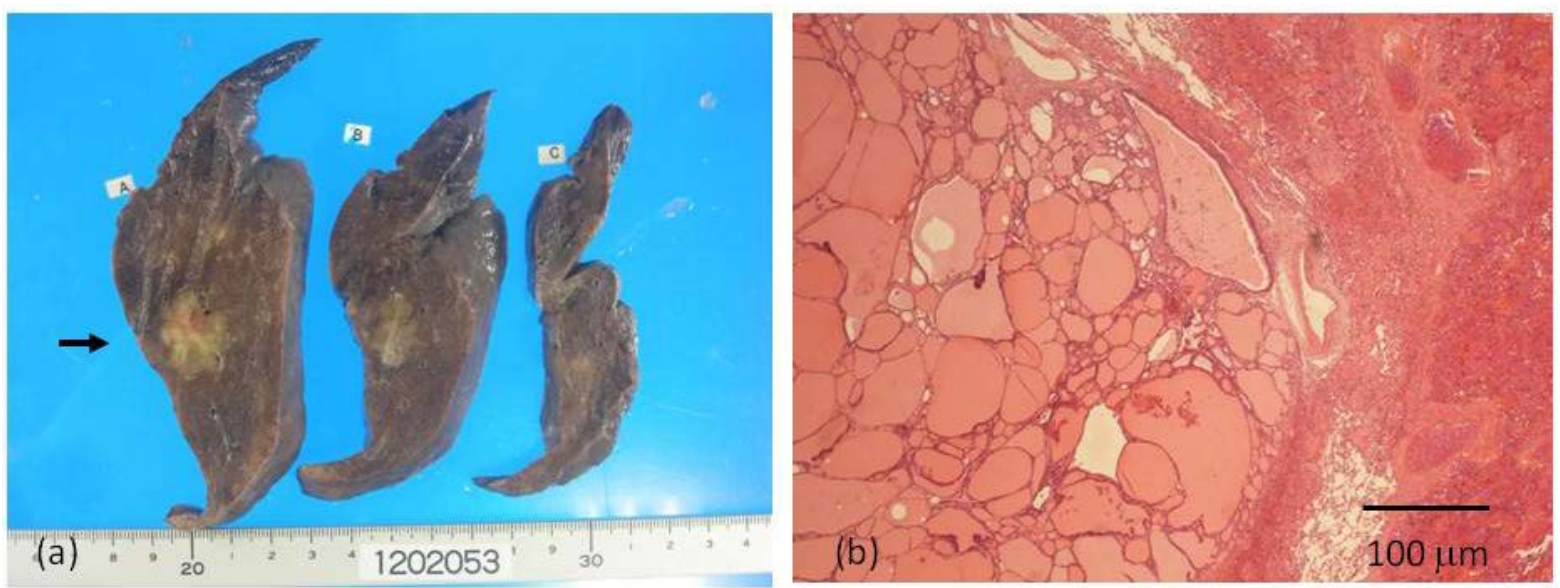

Figure 2. (a) Appearance of the lung tumor after the right lower lobectomy; (b) Histopathologic of the resected tumor revealed thyroid gland with follicular projection; and pathological diagnosis was metastases from thyroid follicular carcinoma (H. E. $\times 200)$.

\section{Discussion}

The lung is one of the most common target organs for metastases from various malignancies. Metastatic lesion manifest radiographically as solitary or multiple nodular patterns. The accurate diagnosis of pulmonary nodules in patients with a history of extrapulmonary malignancy is usually difficult and it is important to proceed to the optimal treatment. The differential diagnoses include primary lung cancer, metastatic lung cancer, and benign tumors (e.g. granulomas and hamartomas). Surgical resection, chemotherapy, radiation, observation, or combined approaches are proposed depending on the origin of the nodules. Histological confirmation was not obtained before surgical resection, but we considered this case as double primary lung cancer or as metastatic lung cancer from the gastrointestinal tract or gynecological organs to improve the patient's chances for long-term survival. We missed the possibility of metastases from thyroid grand because of the long disease-free interval (30 years) from previous diagnosis and treatment of benign adenoma. Resectable metastasis to the lung from thyroid cancer is quite rare [3] [4].

Pulmonary metastases from thyroid cancer usually present as a multiple nodular pattern or miliary pattern disseminated throughout both lungs [3]. Consequently, surgical resection is not considered, but iodine-131 ( $\left.{ }^{131} \mathrm{I}\right)$ therapy is applied after total thyroidectomy, if indicated.

Ríos et al. analyzed prognostic factors for follicular thyroid carcinoma [5]. Their result indicated that there is a 38\% recurrence rate during a 100-month follow-up period. Three percent of patient die from the disease and 3\% have distant metastases and survived. The mean disease free interval is 154 months. The rates of disease-free patients at 5, 10, 15, and 20 years are $71 \%, 58 \%, 58 \%$, and $58 \%$.

Ronga et al. reported that patients with lung metastases from differentiated thyroid carcinoma, young patents $<45$ years of age at diagnosis, and highly ${ }^{131}$ I uptakes by metastases are the most important factors positively affecting survival times [6].

Although the patient was a stage IV octogenarian, bilateral surgical procedures did not affect her prognosis. She remained disease-free even though she avoids total thyroidectomy and radiotherapy. Resection of metastases to the bilateral lungs from thyroid cancer were quite rare.

\section{Conclusion}

We describe a surgical case of pulmonary metastases from follicular thyroid carcinoma. At least empirically, surgery is the treatment of choice for patients with this condition. Resection of metastases to the bilateral lungs from thyroid cancer is quite rare.

\section{Disclosure Statement}

The authors have no financial or other conflict of interest to declare. 


\section{References}

[1] Ulge, Z., Karaman, N., Piskinpasa, S.V., Niksarlioglu, Y.O., Kilickap, S., et al. (2006) Endobronchial Metastasis of Thyroid Follicular Carcinoma. Journal of the National Medical Association, 98, 803-806.

[2] Schlumberger, M. and Leboulleux, S. (2015) Treatment of Distant Metastases from Follicular Cell-Derived Thyroid Cancer. F1000Prime Reports, 7, 22. http://dx.doi.org/10.12703/P7-22

[3] Shigematsu, H., Andou, A., Teramoto, A., Matsuo, K., Oda, W., et al. (2009) Solitary Lung Metastasis Diagnosed 30 Years after Surgery for Thyroid Cancer. The Annals of Thoracic Surgery, 88, 2016-2017. http://dx.doi.org/10.1016/j.athoracsur.2009.04.134

[4] Sakairi, Y., Yasufuku, K., Iyoda, A., Suzuki, M., Nakajima, T., et al. (2008) A Solitary Metastatic Lung Tumor from Thyroid Papillary Carcinoma Diagnosed by Endobronchial Ultrasound-Guided Transbronchial Needle Aspiration (EBUS-TBNA): Report of a Case. Surgery Today, 38, 46-48. http://dx.doi.org/10.1007/s00595-007-3560-2

[5] Ríos, A., Rodríguez, J.M., Ferri, B., Martínez-Barba, E., Torregrosa, N.M., Parrilla, P., et al. (2015) Prognostic Factors of Follicular Thyroid Carcinoma. Endocrinología y Nutrición, 62, 11-18. http://dx.doi.org/10.1016/j.endonu.2014.06.006

[6] Ronga, G., Filesi, M., Montesano, T., Di Nicola, A.D., Pace, C., et al. (2004) Lung Metastases from Differentiated Thyroid Carcinoma. A 40 Years’ Experience. Quarterly Journal of Nuclear Medicine and Molecular, 48, 12-19. 